Wermuth/Ochynski

Strategien an den Devisenmärkten 


\title{
Strategien an den
}

\section{Devisenmärkten}

- Eine Anleitung für die Praxis -

3., völlig überarbeitete und erweiterte Auflage

\author{
von \\ Dieter Wermuth \\ und \\ Walter Ochynski
}

mit einem Geleitwort von

Friedrich W. Menzel 
CIP-Kurztitelaufnahme der Deutschen Bibliothek

Wermuth, Dieter:

Strategien an den Devisenmärkten : e. Anleitung

für d. Praxis / von Dieter Wermuth u. Walter

Ochynski. Mit e. Geleitw. von Friedrich W. Menzel.

- 3., völlig überarb. u. erw. Aufl. - Wiesbaden :

Gabler, 1987.

(Die Bankgeschäfte)

ISBN-13: 978-3-409-34108-0

e-ISBN-13: 978-3-322-86279-2

DOI: $10.1007 / 978-3-322-86279-2$

NE: Ochynski, Walter:

1. Auflage 1983

2., durchgesehene Auflage 1984

3., völlig überarbeitete und erweiterte Auflage 1987

(C) Betriebswirtschaftlicher Verlag Dr. Th. Gabler GmbH, Wiesbaden 1987

Softcover reprint of the hardcover 3rd edition 1987

Das Werk einschließlich aller seiner Teile ist urheberrechtlich geschützt. Jede Verwertung außerhalb der engen Grenzen des Urheberrechtsgesetzes ist ohne Zustimmung des Verlages unzulässig und strafbar. Das gilt insbesondere für Vervielfältigungen, Übersetzungen, Mikroverfilmungen und die Einspeicherung und Verarbeitung in elektronischen Systemen.

ISBN-13: 978-3-409-34108-0 


\section{Inhaltsverzeichnis}

Geleitwort

Seite

Vorwort zur dritten Auflage $\ldots \ldots \ldots \ldots \ldots \ldots \ldots \ldots \ldots \ldots \ldots \ldots \ldots \ldots \ldots$

Vorwort zur ersten Auflage $\ldots \ldots \ldots \ldots \ldots \ldots \ldots \ldots \ldots \ldots \ldots \ldots \ldots \ldots$

A. Kleine Währungsgeschichte $\ldots \ldots \ldots \ldots \ldots \ldots \ldots \ldots \ldots$

1. Goldstandard ...................... 3

1.1 Paritäten ...................... 3

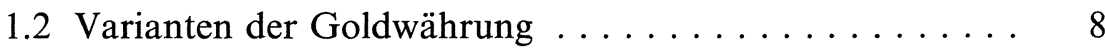

2. Vom Goldstandard zu Bretton-Woods . . . . . . . . . . 11

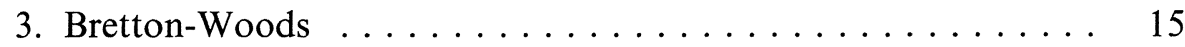

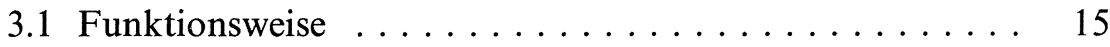

3.2 Große wirtschaftspolitische Erfolge . . . . . . . . . 17

3.3 Gründe für das Scheitern $\ldots \ldots \ldots \ldots \ldots \ldots \ldots . \ldots \ldots$

3.4 Rückzugsgefechte . . . . . . . . . . . . . . . . 19

Kalendarium der modernen Währungsgeschichte .......... 21

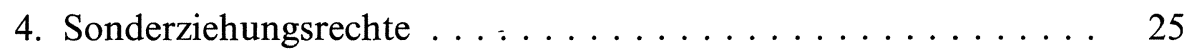

4.1 Gründe für ihre Einführung $\ldots \ldots \ldots \ldots \ldots \ldots 25$

4.2 Definition ................... 26

4.3 Verzinsung der Sonderziehungsrechtskonten beim IWF . . 28

4.4 Sonderziehungsrechte für private Anleger oder Schuldner? 29

Exkurs: Berechnung des Zinses auf Sonderziehungsrecht-Kredite . . 30

5. Flexible Wechselkurse ................. 33

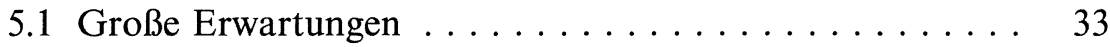

5.2 Starke Ausschläge in den Wechselkursen . . . . . . . . . 34

5.3 Kosten . . . . . . . . . . . . . . . . . . . 37

5.4 Ursachen der Wechselkurszyklen . . . . . . . . . . 38

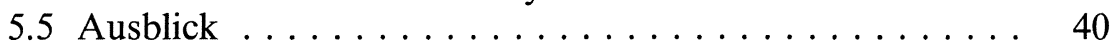

6. Das Europäische Währungssystem $\ldots \ldots \ldots \ldots \ldots \ldots 41$

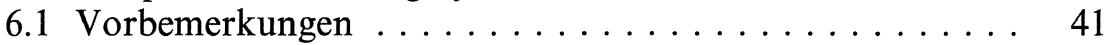

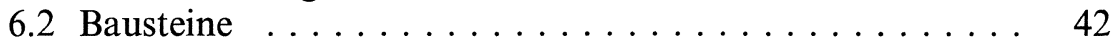

6.2.1 Bilaterale Interventionspunkte . . . . . . . . 42

6.2.2 ECU (European Currency Unit) . . . . . . . . . . 46

6.2 .3 Abweichungsindikator ............. 52

Exkurs: Abweichungsindikator im EWS . . . . . . . . . 52

6.2.4 Saldenausgleich und Kreditmechanismen ...... 53

6.3 Beurteilung .................... 55

B. Grundlagen des Devisenhandels $\ldots \ldots \ldots \ldots \ldots \ldots \ldots \ldots$

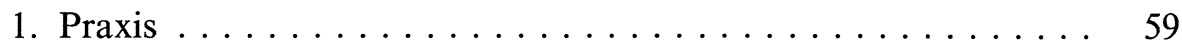


2. Kassamarkt ....................... 61

Exkurs: Quotierung des Händlers $\ldots \ldots \ldots \ldots \ldots \ldots \ldots$

3. Amtliche Devisenkurse . . . . . . . . . . . . . 71

4. Termin- und Swapgeschäfte $\ldots \ldots \ldots \ldots \ldots \ldots \ldots \ldots$

C. Aufgaben des Finanzmanagers $\ldots \ldots \ldots \ldots \ldots \ldots \ldots \ldots$

1. Swapsätze und Zinsdifferenzen $\ldots \ldots \ldots \ldots \ldots \ldots \ldots 87$

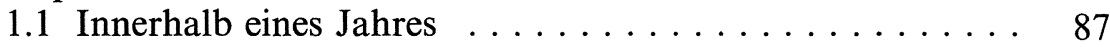

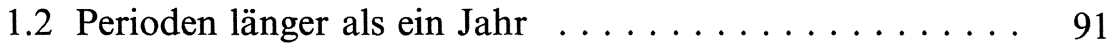

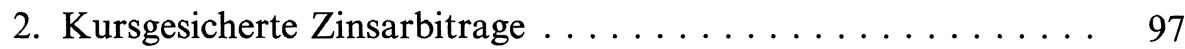

3. Cross Currency Funding $\ldots \ldots \ldots \ldots \ldots \ldots \ldots \ldots$

3.1 Günstigste Anlagewährung . . . . . . . . . . . . 101

3.2 Günstigste Kreditaufnahmewährung . . . . . . . . . 103

Exkurs: Bankers' Acceptances . . . . . . . . . . . . . . . . . . 105

4. Hedging der zukünftigen Zahlungsströme . . . . . . . . . . 109

4.1 Warum Hedging? . . . . . . . . . . . . . . . . . . . 109

4.2 Einführung in die Methode . . . . . . . . . . 111

4.3 Kurssicherung voñ Währungsforderungen . . . . . . . 114

4.4 Kurssicherung von Währungsverbindlichkeiten .... . . . 119

4.5 Absicherung bei Fehlen eines Terminmarktes oder bei Kapitalverkehrskontrollen .............. 121

4.5.1 Der Fall des Cross-Currency Hedging . . . . . . 121

4.5.2 Gegenseitige Wechselkursgarantie . . . . . . . 121

4.5.3 Bankbürgschaften ................ 122

4.5.4 Warenkredit .................. 122

4.5.5 Kompensationsgeschäfte . . . . . . . . . 122

4.5.6 Verkauf von Fremdwährungsforderungen . . . . 122

4.5.7 Hermes Kreditversicherung . . . . . . . . . . . 123

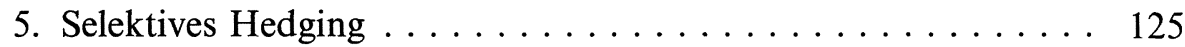

5.1 Hedging bei bereits abgeschlossenen Warengeschäften . . . 125

5.2 Hedging bei buchmäßigen Umrechnungsrisiken . . . . . 125

6. Berechnung der Kurssicherungskosten . . . . . . . . . . 127

7. Umrechnung auf der alten Basis . . . . . . . . . . . . 131

8. Beibehaltung einer offenen Position . . . . . . . . . . . 139

9. Ungesicherte Fremdwährungsanlage oder Fremdwährungsaufnahme ......................... 141

10. Hedging auf den Futures-Märkten . . . . . . . . . . 143

10.1 Grundbegriffe . . . . . . . . . . . . . . . . 145

10.2 Futures-Märkte und Terminmärkte . . . . . . . . . . 147

10.3 Warentermin-Futures . . . . . . . . . . . . . . . . 148 
10.4 Aktien-Index-Futures . . . . . . . . . . . . . . . . . . . 148

10.5 Devisen-Futures . . . . . . . . . . . . . . . . . . . . . 149

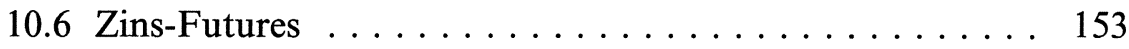

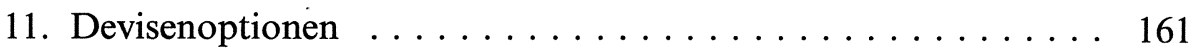

11.1 Wichtige Begriffe $\ldots \ldots \ldots \ldots \ldots \ldots \ldots \ldots \ldots$

11.2 Optionen im Vergleich zu Termingeschäften . . . . . . . . 164

Exkurs: An Börsen gehandelte Devisenoptionen . . . . . . . . . . 165

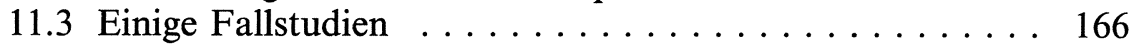

11.3.1 Der Fall eines Anlagenbauers . . . . . . . . . 166

11.3.2 Ein deutscher Importeur von Mineralölprodukten . 166

11.3.3 Ein ausländischer Kunde hat Zahlungsprobleme . . 167

11.3.4 Weitere Fälle, in denen sich der Kauf von

Devisenoptionen empfiehlt ............ 167

11.3.5 Wann lohnt der Verkauf von Devisenoptionen? . . 168

Exkurs: Wie die Volatilität berechnet wird - ein Rechenbeispiel . . 170

11.4 Strategien am Markt für Devisenoptionen . . . . . . . . 173

11.4.1 Die elementaren Strategien . . . . . . . . . . 173

11.4.2 Ein graphischer Vergleich von Termingeschäften und Optionsgeschäften ............. 176

11.4.3 Synthetische Positionen mit Hilfe von Devisenoptionen und Termingeschäften ............ 177

11.4.4 Strategien bei begrenzten DM-Aufwertungserwar-

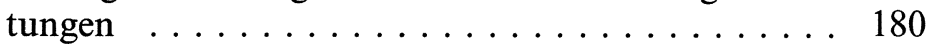

11.4.5 Strategien bei begrenzten DM-Abwertungserwartungen

11.4.6 Strategien, die auf Erwartungen über künftige Wechselkursschwankungen beruhen . . . . . . . . . . 183

12. Zins- und Währungsswaps . . . . . . . . . . . . . . . 187

12.1 Definitionen, Abgrenzungen und ein Einführungsbeispiel . 187

12.2 Beispiele . . . . . . . . . . . . . . . . . . 190

12.2.1 Währungsswaps . . . . . . . . . . . . . . 190

12.2.2 Zinsswaps .................... 192

12.2.3 Beispiel für einen Cocktail-Swap mit drei Währun-

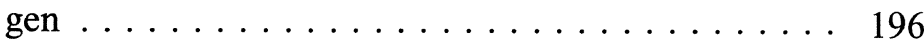

12.2.4 Aktivswaps . . . . . . . . . . . . . . . . 198

12.3 Die Rolle der Banken . . . . . . . . . . . . . . . 201

12.4 Preisfindung ................... 201

13. Terminmarkt-Produkte $\ldots \ldots \ldots \ldots \ldots \ldots \ldots \ldots \ldots$

D. Determinanten der Wechselkurse $\ldots \ldots \ldots \ldots \ldots \ldots \ldots$

1. Rolle der Leistungsbilanz . . . . . . . . . . . . . . . . . . . . 209

Exkurs: Definition der Leistungsbilanz . . . . . . . . . . 211

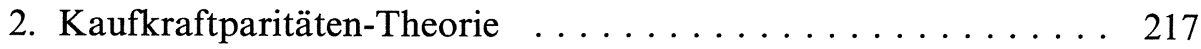

Exkurs: Gewogene und reale Wechselkurse . . . . . . . . . 222 
3. Monetaristischer Ansatz . . . . . . . . . . . . . . . . . . . . 229

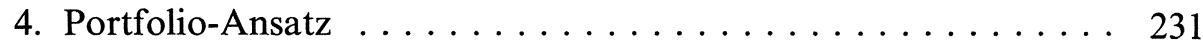

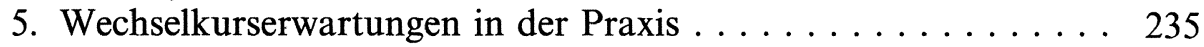

E. Technische Analyse - oder wie sich Trends entdecken und nutzen lassen 243

1. Werkzeuge ....................... 247

1.1 Graphische Analyse . . . . . . . . . . . . . . . . . 247

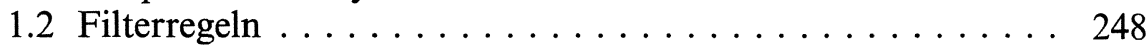

1.3 Gleitende Durchschnitte . . . . . . . . . . . . . . . . . . 249

1.4 Momentum-Regeln . . . . . . . . . . . . . . . 250

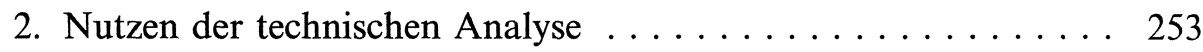

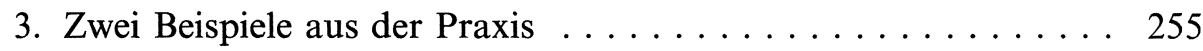

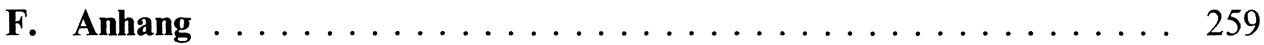

1. Ableitung der Zinsformel für Swapsätze unter einem Jahr . . . . 261

2. Ableitung der Zinsformel für Swapsätze für mehr als ein Jahr . . 265

Verzeichnis der Abbildungen $\quad \ldots \ldots \ldots \ldots \ldots \ldots \ldots \ldots \ldots$

Verzeichnis der Tabellen . . . . . . . . . . . . . . . . . . . . 272

Literaturverzeichnis . . . . . . . . . . . . . . . . . . . . 274

Stichwortverzeichnis . . . . . . . . . . . . . . . . . . 276 


\section{Geleitwort}

Die Weltwirtschaft wächst immer enger zusammen. Der internationale Warenverkehr nimmt Jahr für Jahr rascher zu als der Umsatz im Inland; selbst während der beiden letzten tiefen Rezessionen, von denen nahezu alle Länder betroffen waren, setzte sich dieser Trend fort. Noch augenfälliger ist die rasche Integration der Kapitalmärkte über die Grenzen hinweg. Immer mehr Unternehmen tätigen Direktinvestitionen im Ausland, und immer mehr Anleger gehen dazu über, ihre Portefeuilles nach Instrumenten, Fristen und Währungen zu diversifizieren, wobei sie häufig bessere Ergebnisse erzielen, als wenn sie sich auf das Inland beschränken würden. Dem höheren Ertrag steht allerdings ein höheres Risiko gegenüber, weil Gewinne leicht durch eine gegenläufige Entwicklung der Wechselkurse wieder zunichte gemacht werden können. Die starken Ausschläge am Devisenmarkt, die die Jahre seit dem Zusammenbruch des Systems von Bretton-Woods kennzeichnen, lassen sich nur schwer mit einiger Zuverlässigkeit vorhersagen. Das Schutzbedürfnis ist entsprechend gestiegen, und die Märkte haben darauf mit einer Reihe von neuartigen Instrumenten reagiert. Manche von ihnen sind so jungen Datums, daß sie bislang kaum im Detail bekannt sind.

Ich glaube, daß das vorliegende Buch hier eine Lücke füllen und die Zusammenarbeit zwischen Unternehmen und Banken verbessern wird. Auch Privatpersonen, die Teile ihres Vermögens im Ausland angelegt oder Kredite in fremden Währungen aufgenommen haben, dürften durch die Lektüre des Buches einen nützlichen Überblick erhalten. Für Studenten der Volks- und Betriebswirtschaftslehre und für Praktiker in den Finanzabteilungen international tätiger Unternehmen bietet das Buch ebenfalls eine umfassende Informationsquelle.

Zahlreiche Märkte stecken noch in den Kinderschuhen. Es ist abzusehen, daß einige Kapitel immer wieder ergänzt und umgeschrieben werden müssen. Ich wünsche dem Buch daher zahlreiche Neuauflagen.

Friedrich W. Menzel

Vorstandsmitglied der

Citibank $A G$, Frankfurt am Main 


\section{Die Autoren}

Dieter Wermuth, Jahrgang 1940, war nach kaufmännischer Lehre, Studium in München und Promotion in den USA von 1973 bis 1978 im Stab des Sachverständigenrats zur Begutachtung der gesamtwirtschaftlichen Entwicklung. Seit 1978 ist er bei der Citibank AG in Frankfurt beschäftigt.

Walter Ochynski, geboren 1952, studierte von 1974 bis 1978 Betriebswirtschaftslehre in Köln, wo er 1980 bei Prof. Dr. H.-K. Schneider auf dem Gebiet der Währungspolitik promovierte. Von 1982 bis 1984 war er bei der Citibank AG beschäftigt. Gegenwärtig ist er im Finanzmanagement der Braun AG in Kronberg tätig.

\section{Vorwort zur dritten Auflage}

Die dritte Auflage unterscheidet sich von der zweiten Auflage durch mehrere Änderungen und Ergänzungen:

Kapitel B wurde neu bearbeitet. Mehrere Beispiele vereinfachen den Einstieg in die Technik des Devisenhandels. Eine Methode für die Kurssicherung des Liquiditätsverlustes bei der forward against forward-Arbitrage wurde eingehend dargestellt. Kapitel C weist die meisten Änderungen auf. Neu hinzugekommen ist ein Teil über Terminmarktprodukte. Die Teile über Hedging auf den Futures-Märkten, Devisenoptionen sowie Zins- und Währungsswaps wurden neu konzipiert. In diesen Teilen werden Sachverhalte behandelt, die die größte Entwicklung in den letzten Jahren erfahren haben.

Kleinere Änderungen und Einfügungen finden sich an vielen anderen Stellen. Das Literaturverzeichnis wurde auf den neuesten Stand gebracht.

Wir danken den zahlreichen Lesern dieses Buches für Anregungen und Verbesserungsvorschläge. Besonders verpflichtet sind wir Herrn Jens-Uwe Fischer, der das Manuskript über die Grundlagen des Devisenhandels gelesen und uns auf einige unklare Stellen aufmerksam gemacht hat.

Walter Ochynski

Dieter Wermuth

Frankfurt, im April 1987 


\section{Vorwort zur ersten Auflage}

Dem atemberaubenden Tempo, mit dem sich die Wechselkurse ändern, seit sie nicht mehr an den Dollar gebunden sind, entspricht das Tempo, mit dem an den Devisenmärkten neue Verfahren und Instrumente ersonnen und eingeführt werden. Wir haben mit diesem Buch den Versuch unternommen, einen Überblick über die modernen Devisenmärkte zu geben, immer aus dem Blickwinkel international tätiger Unternehmen. Das Schwergewicht liegt auf Lösungsvorschlägen für Probleme, die sich Finanzmanagern ständig stellen, sobald ihr Unternehmen geschäftlich mit dem Ausland zu tun hat. Die Politik der Kurssicherung entscheidet heute oft wesentlich über den Erfolg.

Würden die Wechselkurse immer den Kaufkraftparitäten folgen, wäre die Arbeit der Finanzmanager leichter. Die starken Schwankungen der Wechselkurse haben uns aber in den letzten Jahren das Gegenteil gelehrt - nur über verhältnismäßig lange Zeiträume bestätigt sich die Regel. Die Risiken für die Unternehmen sind daher groß, groß sind aber auch die Chancen, die sich durch geschickte Strategien nutzen lassen.

Wir beschränken uns nicht nur auf die technische Seite der Probleme, sondern gehen einen Schritt weiter und stellen in leicht verständlicher Sprache das Gerüst der modernen Wechselkurstheorie dar (Kapitel D). Ihr Verständnis erlaubt es, sich ein eigenes Urteil über die künftige Entwicklung der Kurse zu bilden. Wer aber wenig Zeit hat oder nur über unzulängliche Materialquellen verfügt, kann sich bei seinem Agieren am Devisenmarkt verhältnismäßig risikolos auf die Signale verlassen, die ihm die sogenannten technischen Wechselkursmodelle bieten (Kapitel E).

Vieles, was heute an den Devisenmärkten geschieht und künftig geschehen kann, ist leichter verständlich, wenn man weiß, welche alternativen Systeme in früheren Zeiten bereits ausprobiert wurden, was ihre Vorzüge waren und warum sie gescheitert sind. Historische Kenntnisse vermitteln die Gelassenheit, an der es im Tagesgeschäft oft fehlt. In einer kleinen Währungsgeschichte zu Beginn (Kapitel A, Teile 1, 2, 3 und 5) nehmen wir die heutige Diskussion des Goldstandards auf: wird die Welt wieder einmal zu diesem überaus erfolgreichen System zurückkehren? Oder wenigstens zu etwas, das dem Dollarstandard von BrettonWoods entspricht, der ja auch durch niedrige Inflationsraten und rasches Wirtschaftswachstum gekennzeichnet war? Hat das gegenwärtige System des „Managed Floating“ nur Nachteile?

Internationale Institutionen wie der Internationale Währungsfonds gewinnen immer mehr an Bedeutung. Seine Instrumente, beispielsweise die Sonderziehungsrechte, sind nicht nur für die Notenbanken von Interesse. Auch der Finanzmanager eines Unternehmens sollte sie bei seinen Geldaufnahme- und Geldanlageentscheidungen berücksichtigen (Kapitel A, Teil 4).

Mit dem Europäischen Währungssystem gibt es in Europa ein System der stufenflexiblen Wechselkurse. Die Kurse sind nicht für alle Zeiten fest, sie werden immer wieder durch politische Entscheidungen geändert. Worauf der Finanzmanager dabei achten muß und wie er die Bausteine des EWS, das ECU oder den Abweichungsindikator, für seine Ziele nutzen kann, wird ausführlich erläutert (Kapitel A, Teil 6). 
Die Darstellung der Grundlagen des Devisenhandels, wie er in den Devisenabteilungen der Großbanken stattfindet, wird dem Finanzmanager die Zusammenarbeit mit den Devisenhändlern erleichtern (Kapitel B).

Manche Aufgaben des Finanzmanagers lassen sich schematisieren, Lösungen mit der größten Genauigkeit ausrechnen. Welche Kurssicherungspolitik soll ein Finanzmanager betreiben? Wie kann er am günstigsten eine Währung anlegen? Wo kann er sich am billigsten Liquidität beschaffen? Wie kann er Devisentermingeschäfte abschließen, die er später nach seinem Belieben ausführen kann, aber nicht muß? Welche Chancen bieten die Märkte für Financial Futures? Anhand von Rechenbeispielen werden Musterfälle durchgespielt (Kapitel C).

Wir möchten an dieser Stelle unseren Kollegen, den Mitarbeitern der Citibank, für ihre Hilfe beim Schreiben des Buches danken. Es ist entstanden aus der Überlegung, den Teilnehmern an den Devisenseminaren, die die Bank regelmäßig veranstaltet, eine Art Nachschlagewerk in die Hand zu geben. Vieles, was in den Seminaren nur gestreift werden kann, wurde in größerer Ausführlichkeit dargestellt. Insbesondere gilt daher unser Dank Friedrich Menzel vom Vorstand der Citibank, der die Seminare seit Jahren leitet. Philip D. Sherman, Vorstandsvorsitzender der Bank, hat uns ermutigt, unser Manuskript zu veröffentlichen. Von Rainer Streb stammen einige der Rechenbeispiele. Hans Bach, Heiner Flassbeck, Udo Gerlach, Gerhard Jänsch, Dr. Norbert Kleinheyer, Peter Klotzsch, Dietmar Kordel, Helmut Predikant, Peter Reubelt, Hans Ritz, Jürg Schaufelberger, Dr. Diethard B. Simmert, Klaus Weber und Elke Ward-Smith haben verschiedene Teile der Arbeit mit uns diskutiert, haben uns beim Sammeln des Materials geholfen oder haben den Text kritisch durchgelesen. Ihnen allen danken wir herzlich. Für Fehler oder Ungenauigkeiten, die verblieben sind, haben wir selbstverständlich geradezustehen.

Das Manuskript wurde von Doris Schäffer auf ihre gewohnt professionelle Art in eine lesbare und übersichtliche Form gebracht. Ohne ihr Engagement hätte sich die Veröffentlichung vor allem wegen der zahlreichen Tabellen und Schaubilder erheblich verzögert. 